\title{
Single nucleotide polymorphisms (SNPs) in coding regions of canine dopamine- and serotonin-related genes Jørn Våge* and Frode Lingaas
}

\author{
Address: Division of Genetics, Department of Basic Sciences and Aquatic Medicine, Norwegian School of Veterinary Science, Oslo, Norway \\ Email: Jørn Våge* - jorn.vage@veths.no; Frode Lingaas - frode.lingaas@veths.no \\ * Corresponding author
}

Published: 28 January 2008

BMC Genetics 2008, 9:10 doi:10.1/86/147|-2156-9-10
Received: 18 June 2007

Accepted: 28 January 2008

This article is available from: http://www.biomedcentral.com/I47/-2/56/9//0

(C) 2008 Våge and Lingaas; licensee BioMed Central Ltd.

This is an Open Access article distributed under the terms of the Creative Commons Attribution License (http://creativecommons.org/licenses/by/2.0), which permits unrestricted use, distribution, and reproduction in any medium, provided the original work is properly cited.

\begin{abstract}
Background: Polymorphism in genes of regulating enzymes, transporters and receptors of the neurotransmitters of the central nervous system have been associated with altered behaviour, and single nucleotide polymorphisms (SNPs) represent the most frequent type of genetic variation. The serotonin and dopamine signalling systems have a central influence on different behavioural phenotypes, both of invertebrates and vertebrates, and this study was undertaken in order to explore genetic variation that may be associated with variation in behaviour.
\end{abstract}

Results: Single nucleotide polymorphisms in canine genes related to behaviour were identified by individually sequencing eight dogs (Canis familiaris) of different breeds. Eighteen genes from the dopamine and the serotonin systems were screened, revealing 34 SNPs distributed in 14 of the 18 selected genes. A total of 24,895 bp coding sequence was sequenced yielding an average frequency of one SNP per 732 bp (I/732). A total of II non-synonymous SNPs (nsSNPs), which may be involved in alteration of protein function, were detected. Of these II nsSNPs, six resulted in a substitution of amino acid residue with concomitant change in structural parameters.

Conclusion: We have identified a number of coding SNPs in behaviour-related genes, several of which change the amino acids of the proteins. Some of the canine SNPs exist in codons that are evolutionary conserved between five compared species, and predictions indicate that they may have a functional effect on the protein. The reported coding SNP frequency of the studied genes falls within the range of SNP frequencies reported earlier in the dog and other mammalian species. Novel SNPs are presented and the results show a significant genetic variation in expressed sequences in this group of genes. The results can contribute to an improved understanding of the genetics of behaviour.

\section{Background}

Neurotransmitters of the central nervous system (CNS) indisputably are important for modulation of the dispersed behaviour seen in both man and animals. Polymorphism in genes of regulating enzymes, transporters and receptors have been associated with altered behaviour [1-3]. Single nucleotide polymorphisms (SNPs) represent the most frequent type of genetic variation in human populations. Non-synonymous SNPs (nsSNPs) comprise a group of SNPs that, together with SNPs in regulatory regions, are believed to have the highest impact on phenotype [4]. 
The genetic basis of behaviour has been explored within a wide range of genes representing neurotransmitters and signalling molecules, and the vast majority of work has been performed on monoamine systems. The serotonin and dopamine signalling systems are central to different behavioural phenotypes, both of invertebrates and vertebrates [5-9]. Marino et al. [10] reported that defects in the noradrenergic system have been implicated in many mood, cognitive and neurological disorders that manifest abnormal social behaviour, and demonstrate that dopamine $\beta$-hydroxylase knock-out (Dbh-/-) mice are deficient in social discrimination and lack isolation-induced aggression. Monoamine oxidase (MAO) A and B play an important role in regulating levels of biogenic amines. Whereas MAOA preferentially oxidises the biogenic amines serotonin, norepinephrine and epinephrine, MAOB preferentially oxidises phenylethylamine and benzylamine. Dopamine, tyramine and tryptamine are common substrates for both forms [11]. MAO A/B double knock-out mice showed increased brain levels of several biogenic amines, and chase/escape and anxiety-like behaviour, suggesting that alterations of monoamine levels are implicated in a unique biochemical and behavioural phenotype [12]. Polymorphism within receptor genes of dopamine and serotonin are associated with a variety of human psychiatric disorders [13-16], and knockout models of $5 H T R 1 B$ produce a deviant mice behaviour [17].

The knowledge of genes associated to behavioural traits is increasing. Characterisations of these genes and identification of closely linked SNPs and microsatellites make it possible to study the segregation of behaviour-associated haplotypes and to learn more about the genetic contribution to canine behaviour. SNPs as abundant polymorphisms scattered over the genomes are important tools for detailed mapping [18]. Beside their value as markers, some of these variations represent polymorphisms with functional effects. Descriptions of genetic variation in expressed sequences and changes in protein sequence may contribute to reveal the causes of differences in behavioural phenotypes.

The large number of canine breeds exhibits an extreme between-breed variation in traits like size, colour, conformation and behaviour. For many of these breeds, behavioural characteristics represent an important part of the breed definition and description. Certain behavioural phenotypes are associated with specific breeds as a result of long-term, systematic selection and limited genetic variation. In a behavioural context, dog breeds are evidence for the considerable impact of genetics on behavioural traits. They are therefore valuable models for genetic studies aimed at revealing basic biological knowledge of genetic regulation of behavioural traits. This can be effi- ciently performed through crossbreeding and backcrosses of these isolates with strong between-breed contrasts in specific behaviours.

Some recent publications characterise polymorphisms in the canine dopamine and serotonin gene families [1923 ], but the number of reported SNPs in coding sequence of behaviour-related genes from dogs is still low. A better knowledge of genetic variation in these genes will be important for an improved understanding of the genetic influence on behaviour in both animals and humans. This study presents novel SNPs in coding sequences of canine serotonin- and dopamine-related genes.

\section{Results}

Sequencing a total number of 24,895 bp coding DNA in each of eight dogs of different breeds revealed a total of 34 SNPs, 30 of them not earlier reported, distributed in 14 of the 18 selected genes (Table 1). SNPs were identified in five genes in the dopamine pathway, in one gene related to synthesis of norepinephrine and in nine genes in the serotonin pathway (Table 2).

The 34 SNPs comprised 23 synonymous and 11 non-synonymous, with the predicted changes in amino acids as described in Table 2 (for flanking nucleotide sequences see Additional file 1). Of the 11 nsSNPs, three held the first position, seven held the second position and one held the third position of the codon. Categorisation of the SNPs according to nucleotide substitution gave 31(91\%) transitions and $3(9 \%)$ transversions, the transversions all being nsSNPs. Six of the $11 \mathrm{nsSNPs}$ resulted in a substitution of amino acid residue with a concomitant shift of class dependent on $\mathrm{R}$ group, and change in structural parameters (Table 3). Looking at conservation of amino acids in the location of detected nsSNPs we found that across five mammalian species (Homo sapiens, Pan troglodytes, Canis familiaris, Mus musculus and Rattus norvegius, at HomoloGene, [24]) four of the sites were reported invariant and seven reported variable (Table 3). Part of the alignment of the protein products from these five species, containing the canine nsSNPs (ClustalW, [25]) are shown in Figure 1.

The potential functional effects of the 11 identified substitutions caused by the nsSNPs were explored using the software PolyPhen [4], designed to predict functional effects of amino acid substitutions (in humans). The predictions are classified as unknown, benign, possibly damaging and probably damaging. The results showed that the effect of the amino acid substitution was predicted to change the function in three of the residues (possibly/ probably damaging) and was classified as benign in seven of the substitutions. In one of the substitutions the effect was unknown (Table 3). 
Table I: List of genes included in the study, with sequence screened and SNP frequency

\begin{tabular}{|c|c|c|c|c|}
\hline Gene & Gene symbol & Gene product category/function* & Bp screened & SNP/bp \\
\hline \multicolumn{5}{|l|}{ Dopamine related genes } \\
\hline Tyrosine hydroxylase & $\mathrm{TH}$ & Presynaptic form. of DOPA & 1488 & I/I488 \\
\hline Dopamine transporter & SLC6A3 & Synaptic clearance of Dopamine & $188 \mid$ & $\mathrm{I} / 470$ \\
\hline Dopamine receptor $\mathrm{DI}$ & DRDI & G protein-coupled receptor & $|34|$ & $1 / 335$ \\
\hline Dopamine receptor D2 & $D R D 2$ & G protein-coupled receptor & 1332 & $1 / 666$ \\
\hline Dopamine receptor D3 & DRD3 & G protein-coupled receptor & 1344 & $1 / 336$ \\
\hline \multicolumn{5}{|l|}{ Norepinephrine related gene } \\
\hline Dopamine beta hydroxylase & DBH & Presynaptic form. of norepinephrine & 1839 & $1 / 306$ \\
\hline \multicolumn{5}{|l|}{ Serotonin related genes } \\
\hline 5-hydroxytryptamine receptor IA & HTRIA & G protein-coupled receptor & 1272 & $1 / 1272$ \\
\hline 5-hydroxytryptamine receptor IB & $H T R I B$ & G protein-coupled receptor & 1170 & $\mathrm{l} / 585$ \\
\hline 5-hydroxytryptamine receptor ID & HTRID & G protein-coupled receptor & 1134 & $1 / 283$ \\
\hline 5-hydroxytryptamine receptor IE & HTRIE & $\mathrm{G}$ protein-coupled receptor & 1098 & $1 / 1098$ \\
\hline 5-hydroxytryptamine receptor IF & HTRIF & G protein-coupled receptor & 1101 & $1 / 1101$ \\
\hline 5 -hydroxytryptamine receptor $2 \mathrm{~A}$ & HTR2A & G protein-coupled receptor & 1414 & 0 \\
\hline 5-hydroxytryptamine receptor $2 B$ & HTR2B & G protein-coupled receptor & 1305 & $1 / 652$ \\
\hline 5-hydroxytryptamine receptor $2 \mathrm{C}$ & HTR2C & G protein-coupled receptor & 1377 & I/I377 \\
\hline 5 -hydroxytryptamine receptor $3 A$ & HTR3A & Ligand-gated ion channel & 1452 & I/I452 \\
\hline 5-hydroxytryptamine receptor 4 & HTR4 & G protein-coupled receptor & 1167 & 0 \\
\hline Serotonin transporter & SLC6A4 & Synaptic clearance of Serotonin & 1845 & 0 \\
\hline Tryptophan hydroxylase I & $\mathrm{TPHI}$ & Presynaptic form. of hydroxytryptophan $(5 \mathrm{HT})$ & 1335 & 0 \\
\hline Sum of bp screened & & & 24895 & \\
\hline No. of SNPs detected & & & 34 & \\
\hline Frequency of SNPs (snp/bp) & & & $1 / 732$ & \\
\hline Synonym $(n=23)$ & & & $1 / 1082$ & \\
\hline Non-synonym $(n=1 \mathrm{I})$ & & & $\mathrm{I} / 2263$ & \\
\hline
\end{tabular}

* As defined at [35]

\section{Discussion}

Being the most frequent variation of DNA, SNPs represent important causes of transcript variation. The identification and closer study of these polymorphisms are important for the assignment of the genetic contribution to different phenotypes. This study describes SNPs in genes from neurotransmitter systems that are reported to be related to different behavioural phenotypes.

SNP frequencies show a considerable variation between species [18,26-28], and Lindblad-Toh et al. [29] presents in $\operatorname{dog}$ a between-breed SNP frequency of $\sim 1 / 900 \mathrm{bp}$ based on shotgun sequence data from each of nine diverse breeds compared to the boxer genome. The SNP frequency detected in our study $(1 / 732$, see Table 1$)$, where a higher number of chromosomes are compared, falls within this range. We are not aware of prior studies reporting SNP frequency of coding sequences from a number of canine genes.
In our study we observed a $\sim 2.5$ times higher number of SNPs in dopamine-related genes compared to serotoninrelated genes. In the group of dopamine-related genes we observed 15 SNPs/7,386 bp sequenced (1/492), while 12 SNPs/14,218 bp (1/1184) were observed in the group of serotonin-related genes $(\mathrm{p}<0.05)$. This may indicate a greater conservation or a greater similarity in the gene structure of the serotonin-related genes compared to the group of dopamine-related genes. The two gene sets represent genes with similar function related to the respective neurotransmitters. The G-coupled receptors are, however, more numerously represented among the serotonin related genes (Table 1 ). One gene, HTR3A is a ligandgated ion channel and is kept out of the analysis.

Since the completion of the sequence for several genomes, there has been an increased focus on functional polymorphism. Databases containing huge numbers of SNPs are now available for the research community. Besides outlining genome architecture with gene location and descrip- 
Table 2: SNPs according to position, function, predicted changes in amino acid and novelty.

\begin{tabular}{|c|c|c|c|c|c|c|c|c|c|}
\hline Gene & CFA* & Region & Allel & Function ${ }^{\dagger}$ & $\begin{array}{l}\text { Codon } \\
\text { position }\end{array}$ & Protein residue $\ddagger$ & $\begin{array}{l}\text { Aa } \\
\text { position }\end{array}$ & Novelty of SNP§ & Reference sequencell \\
\hline $\mathrm{TH}$ & 18 & EX 13 & $\mathrm{C} / \mathrm{T}$ & syn & 3 & Asp [D] & 482 & Novel & $\begin{array}{l}\text { [GenBank: } \\
\text { NM 001002966.1] }\end{array}$ \\
\hline \multirow[t]{4}{*}{ SLC6A3 } & 34 & EX 9 & $\mathrm{~A} / \mathrm{G}$ & syn & 3 & Gly [G] & 418 & Novel & [GenBank: XM 846543.I] \\
\hline & & EX 10 & $\mathrm{C} / \mathrm{T}$ & syn & 3 & Asp [D] & 468 & Publ. - rs23877306 & [GenBank: XM 846543.I] \\
\hline & & EX 10 & $\mathrm{~A} / \mathrm{G}$ & syn & 3 & $\mathrm{Ala}[\mathrm{A}]$ & 471 & Novel & [GenBank: XM 846543.I] \\
\hline & & EX II & $\mathrm{C} / \mathrm{T}$ & syn & 3 & Asp [D] & 498 & Novel & [GenBank: XM 846543.1] \\
\hline \multirow[t]{4}{*}{ DRD I } & 4 & EX I & $\mathrm{A} / \mathrm{G}$ & syn & 3 & Ala $[\mathrm{A}]$ & 84 & Novel & [GenBank: XM 546227.2] \\
\hline & & EXI & $\mathrm{C} / \mathrm{T}$ & syn & 3 & Gly [G] & 88 & Novel & [GenBank: XM 546227.2] \\
\hline & & EX I & $\mathrm{C} / \mathrm{T}$ & syn & 3 & Val [V] & 236 & Novel & [GenBank: XM 546227.2] \\
\hline & & EXI & $\mathrm{C} / \mathrm{T}$ & ns & 2 & $\operatorname{Thr}[\mathrm{T}] / \mathrm{Met}[\mathrm{M}]$ & 354 & Novel & [GenBank: XM 546227.2] \\
\hline \multirow[t]{2}{*}{$D R D 2$} & 5 & EX 2 & $\mathrm{C} / \mathrm{T}$ & syn & 3 & Asn $[\mathrm{N}]$ & 23 & Novel & $\begin{array}{l}\text { [GenBank: } \\
\text { NM } 001003 \mid 10.1]\end{array}$ \\
\hline & & EX 2 & $\mathrm{C} / \mathrm{T}$ & syn & 3 & Ala $[\mathrm{A}]$ & 77 & Novel & $\begin{array}{l}\text { [GenBank: } \\
\text { NM } 001003110.1]\end{array}$ \\
\hline \multirow[t]{4}{*}{ DRD3 } & 33 & EX 4 & $\mathrm{C} / \mathrm{T}$ & syn & 3 & Cys [C] & 231 & Novel & [GenBank: XM 545I 06.2] \\
\hline & & EX 6 & $\mathrm{~A} / \mathrm{G}$ & ns & 2 & Gln $[\mathrm{Q}] / \operatorname{Arg}[\mathrm{R}]$ & 294 & Novel & [GenBank: XM 545|06.2] \\
\hline & & EX 6 & $\mathrm{C} / \mathrm{T}$ & ns & 1 & Leu $[$ L]/Phe $[\mathrm{F}]$ & 341 & Novel & [GenBank: XM 545I06.2] \\
\hline & & EX 7 & $\mathrm{C} / \mathrm{T}$ & syn & 3 & Cys [C] & 402 & Novel & [GenBank: XM 545I06.2] \\
\hline \multirow[t]{6}{*}{$D B H$} & 9 & EXI & $A / G$ & syn & 3 & $\operatorname{Thr}[\mathrm{T}]$ & 17 & Novel & $\begin{array}{l}\text { [GenBank: } \\
\text { NM 001005263.1] }\end{array}$ \\
\hline & & EX 2 & $A / G$ & syn & 3 & Gln [Q] & 121 & Novel & $\begin{array}{l}\text { [GenBank: } \\
\text { NM 001005263.1] }\end{array}$ \\
\hline & & EX 3 & $\mathrm{C} / \mathrm{T}$ & syn & 3 & $\operatorname{Tyr}[\mathrm{Y}]$ & 163 & Novel & $\begin{array}{l}\text { [GenBank: } \\
\text { NM 001005263.I] }\end{array}$ \\
\hline & & EX 4 & $\mathrm{~A} / \mathrm{C}$ & ns & 3 & Lys $[K] / A s n[N]$ & 263 & Novel & $\begin{array}{l}\text { [GenBank: } \\
\text { NM 001005263.1] }\end{array}$ \\
\hline & & EX 4 & $\mathrm{C} / \mathrm{T}$ & syn & 3 & Gly [G] & 297 & Novel & $\begin{array}{l}\text { [GenBank: } \\
\text { NM 001005263.1] }\end{array}$ \\
\hline & & $\mathrm{EX} 12$ & $A / G$ & syn & 3 & Gly [G] & 622 & Novel & $\begin{array}{l}\text { [GenBank: } \\
\text { NM 001005263.1] }\end{array}$ \\
\hline HTRIA & 2 & EXI & $\mathrm{A} / \mathrm{C}$ & ns & 1 & Lys [K]/Gln [Q] & 270 & Publ. - rs22855024 & [GenBank: XM 544358.I] \\
\hline \multirow[t]{2}{*}{$H T R I B$} & 12 & EXI & $\mathrm{A} / \mathrm{C}$ & ns & 1 & Ile [I]/Leu [L] & 53 & Novel & $\begin{array}{l}\text { [GenBank: } \\
\text { NM 001006948.I] }\end{array}$ \\
\hline & & EXI & $A / G$ & syn & 3 & Pro $[P]$ & 82 & Novel & $\begin{array}{l}\text { [GenBank: } \\
\text { NM 001006948.I] }\end{array}$ \\
\hline \multirow[t]{4}{*}{ HTRID } & 2 & EXI & $\mathrm{C} / \mathrm{T}$ & ns & 2 & Ala $[\mathrm{A}] / \mathrm{Val}[\mathrm{V}]$ & 97 & Novel & $\begin{array}{l}\text { [GenBank: } \\
\text { NM 001003280.1] }\end{array}$ \\
\hline & & EXI & $\mathrm{C} / \mathrm{T}$ & syn & 3 & $\operatorname{Tyr}[\mathrm{Y}]$ & 221 & Novel & $\begin{array}{l}\text { [GenBank: } \\
\text { NM 001003280.1] }\end{array}$ \\
\hline & & EXI & $A / G$ & ns & 2 & Glu [E]/Gly [G] & 263 & Publ. - rs22791523 & $\begin{array}{l}\text { [GenBank: } \\
\text { NM 001003280.I] }\end{array}$ \\
\hline & & EXI & $\mathrm{C} / \mathrm{T}$ & syn & 3 & $\mathrm{His}[\mathrm{H}]$ & 372 & Novel & $\begin{array}{l}\text { [GenBank: } \\
\text { NM 001003280.1] }\end{array}$ \\
\hline HTRIE & 12 & EXI & $\mathrm{C} / \mathrm{T}$ & syn & 1 & Leu [L] & 39 & Novel & [GenBank: XM 539028.I] \\
\hline HTRIF & 31 & EXI & $\mathrm{C} / \mathrm{T}$ & syn & 3 & $\operatorname{Arg}[R]$ & 162 & Publ. - rs9250875 & $\begin{array}{l}\text { [Ensembl: } \\
\text { ENSCAFT000000I24I7] }\end{array}$ \\
\hline \multirow[t]{2}{*}{ HTR2B } & 25 & EXI & $\mathrm{C} / \mathrm{T}$ & ns & 2 & Thr $[\mathrm{T}] / / \mathrm{lle}[\mathrm{I}]$ & 88 & Novel & $\begin{array}{l}\text { [GenBank: } \\
\text { NM 001024633.1] }\end{array}$ \\
\hline & & EX 3 & $\mathrm{C} / \mathrm{T}$ & ns & 2 & Ala $[\mathrm{A}] / \mathrm{Val}[\mathrm{V}]$ & 431 & Novel & $\begin{array}{l}\text { [GenBank: } \\
\text { NM 001024633.I] }\end{array}$ \\
\hline HTR2C & $x$ & EX 6 & $A / G$ & syn & 3 & Pro $[P]$ & 280 & Novel & $\begin{array}{l}\text { [GenBank: } \\
\text { NM 001006648.I] }\end{array}$ \\
\hline HTR3A & 5 & EX 2 & $\mathrm{C} / \mathrm{T}$ & ns & 2 & $\operatorname{Thr}[\mathrm{T}] /$ Met $[\mathrm{M}]$ & 64 & Novel & [GenBank: XM 5465I7.2] \\
\hline
\end{tabular}

* Chromosome number, Canis familiaris.

† Synonymous SNP (syn) and non-synonymous SNP (ns)

$\ddagger$ Listed ambiguity is decided by the respective allel in column 4 .

$\$$ Novelty of SNPs explored by blasting the dbSNP (Dog_9615) at [36]. Reference no. given for corresponding published SNP.

|| Canine sequence/predicted sequence, for defining SNPs position in protein. Given as GenBank or Ensemble accession number. 
Table 3: Shift of residues with change of class according to $\mathbf{R}$ groups, conservation across species and PolyPhen predictions.

\begin{tabular}{|c|c|c|c|c|c|}
\hline Gene & Protein residue* & Aa position & $\begin{array}{l}\text { Change in class according to } \mathbf{R} \\
\text { group }^{\dagger}\end{array}$ & $\begin{array}{l}\text { Residue conservation across } 5 \\
\text { species } \ddagger\end{array}$ & PolyPhen prediction§ \\
\hline DRD I & $\operatorname{Thr}[\mathrm{T}] /$ Met $[\mathrm{M}]$ & 354 & No change & Invariant & Possibly damaging \\
\hline \multirow[t]{2}{*}{ DRD3 } & $\mathrm{G} \ln [\mathrm{Q}] / \operatorname{Arg}[\mathrm{R}]$ & 294 & $\begin{array}{l}\text { Polar, uncharged/positively } \\
\text { charged }\end{array}$ & Variable & Benign \\
\hline & Leu $[\mathrm{L}] /$ Phe $[\mathrm{F}]$ & 341 & Nonpolar, aliphatic/aromatic & Invariant & Benign \\
\hline DBH & Lys $[\mathrm{K}] /$ Asn $[\mathrm{N}]$ & 263 & $\begin{array}{l}\text { Positively charged/polar, } \\
\text { uncharged }\end{array}$ & Variable & Benign \\
\hline HTRIA & Lys [K]/Gln [Q] & 270 & $\begin{array}{l}\text { Positively charged/polar, } \\
\text { uncharged }\end{array}$ & Variable & Benign \\
\hline$H T R I B$ & Ile $[\mathrm{I}] /$ Leu $[\mathrm{L}]$ & 53 & No change & Variable & Benign \\
\hline \multirow[t]{2}{*}{ HTRID } & Ala $[\mathrm{A}] / \mathrm{Val}[\mathrm{V}]$ & 97 & No change & Invariant & Benign \\
\hline & Glu [E]/Gly [G] & 263 & $\begin{array}{l}\text { Negatively charged/Nonpolar, } \\
\text { aliphatic }\end{array}$ & Variable & Benign \\
\hline \multirow[t]{2}{*}{ HTR2B } & Thr [T]/lle [l] & 88 & $\begin{array}{l}\text { Polar, uncharged/nonpolar, } \\
\text { aliphatic }\end{array}$ & Invariant & Probably damaging \\
\hline & $\mathrm{Ala}[\mathrm{A}] / \mathrm{Val}[\mathrm{V}]$ & 431 & No change & Variable & Predicted to be unknown \\
\hline HTR3A & $\operatorname{Thr}[\mathrm{T}] /$ Met $[\mathrm{M}]$ & 64 & No change & Variable & Possibly damaging \\
\hline
\end{tabular}

\footnotetext{
* Changes according to Table 2, shift in residues as result of different alleles of SNPs.

† Changes according to shift of residues in column 2, respectively. Classes according to R groups as described by [37].

$¥$ Residue variation across five mammalian species.

$\S$ Prediction of a possible damaging effect of the amino acid substitutions caused by the nsSNPs, performed with PolyPhen [4].
}

tion of polymorphisms, one of the major challenges is to infer the functional implications of these variations. It has been estimated that $20 \%$ of common human nsSNPs damage the protein [30]. A large database for identification of human nsSNPs with potential impact on disease (PolyDoms, [31]) uses two sequence homology-based tools, SIFT [32] and PolyPhen [4], to predict the potential impact of nsSNP on protein function. Among the structural parameters analysed in PolyPhen for assessing a possible damaging effect of amino acid substitutions are properties in relation to changes of hydrophobicity and electrostatic charge, as well as protein solubility and compatibility of amino acid substitutions in homologous proteins. The changes of R-group classes seen in six of the substitutions in our study (Table 3 ) represent a change in such structural parameters. When inferring about the effect of the predicted amino acid substitutions it can be useful to combine data describing biochemical properties of residues, with knowledge of the conservation across species. Table 3 shows that four residues are evolutionary conserved between the five compared species. Of these, two also experience a change in class of R-group. Presumably one would expect these two substitutions to be the ones most likely to cause functional changes in the protein

\section{Conclusion}

We have identified a number of coding SNPs in behaviour-related genes, several of which change the amino acids of the proteins. Some of the canine SNPs exist in codons that are evolutionary conserved between five compared species, and predictions indicate that they may have a functional effect on the protein. The reported coding SNP frequency of the studied genes falls within the range of SNP frequencies reported earlier in the dog and other mammalian species. Novel SNPs are presented and the results show a significant genetic variation in expressed sequences in this group of genes. The results can contribute to an improved understanding of the genetics of behaviour.

\section{Methods \\ Materials}

Blood samples were collected from eight dogs of eight different breeds - rottweiler, Labrador retriever, Newfoundland, golden retriever, English setter, boxer, Norwegian lundehund and German shepherd. All dogs were healthy pets visiting the veterinary clinic for routine control.

\section{DNA isolation}

DNA was isolated from $10 \mathrm{ml}$ of EDTA-blood by the phenol-chloroform method [33]. DNA was aliquoted and stored at $-20^{\circ} \mathrm{C}$.

\section{Identification of genomic sequences}

The initial identification of relevant canine sequence was performed using comparative genomics, facilitated through the high degree of similarity between human and canine genomes [28,29]. Published human and canine sequences from NCBI and ENSEMBLE were aligned and 


\begin{tabular}{|c|c|c|c|}
\hline \multirow[t]{5}{*}{ DRD 1} & Human & LCPATNNAIETVS INNNGAAMF SSHHEPRGS ISKECNLVYLIPHAVGSSE & 399 \\
\hline & Dog & LCP TĪNNA IETVS INNNGAVVF SSHHEPRGS ISKDCNLVYLIPHAVGSSE & 399 \\
\hline & Mouse & LCP TĪNNAIETVS INNNGAVMF SSHHEPRGS ISKDCNLVYLIPHAVGSSE & 399 \\
\hline & Rat & LCPTİNNAIETVS INNNGAVVF SSHHEPRGSISKDCNLVYLIPHAVGSSE & 399 \\
\hline & & 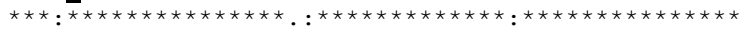 & \\
\hline \multirow{6}{*}{ DRD 3} & Human & 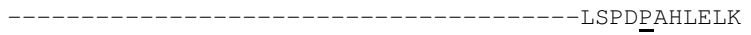 & 253 \\
\hline & Chimp & 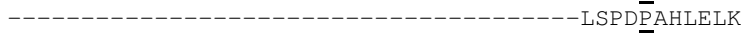 & 253 \\
\hline & Dog & RQF S IRDRFP SDATGQMEHSEDKRYPQKCQDP LLSQLQP LSP SQ̄AHMELK & 300 \\
\hline & Mouse & RQF S IRARF LSDATGQMEHIEDKPYPQKCQDP LLSHLQP LSP GQ̄THGELK & 300 \\
\hline & Rat & RQF S IRARF LSDATGQMEHIEDKQYPQKCQDP LLSHLQPP SP GQ̄THGGLK & 300 \\
\hline & & $\star \star{ }^{-}::^{\star} \quad \star \star$ & \\
\hline \multirow[t]{6}{*}{ DRD 3} & Human & RYYSICQDTALGGPGFQERGGELKREEKTRNSLSPTIAPKLSLEVRKLSN & 303 \\
\hline & Chimp & RYYSICQDTALGGPGEQERGGELKREEKTRNSLSPTIAPKL̄SLEVRKLSN & 303 \\
\hline & Dog & RYYSICQDTALGQPGFQEAGGELKREGRTRNSLSP TMAPKL̈SLEVRKLSN & 350 \\
\hline & Mouse & RYYSICQDTALRHPNFEGGGG-MSQVERTRNSLSP TMAPKL̄SLEVRKLSN & 349 \\
\hline & Rat & RYYSICQDTALRHPSLEGGAG-MSPVERTRNSLSP TMAPKL̈SLEVRKLSN & 349 \\
\hline & & 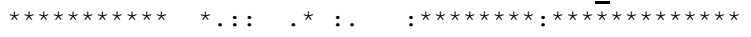 & \\
\hline \multirow[t]{5}{*}{$\mathrm{DBH}$} & Human & IKYEP IVTKGNEALVHHMEVFQCAPEMDSVP HF SGPCDSKMKPDRLNYCR & 282 \\
\hline & Chimp & IKYEP IVTKGNEALVHHMEVFQCA $\bar{P} E L D S V P H F$ S GP CD SKMKPDRLNYCR & 296 \\
\hline & Mouse & IMYEAIVTEGNEALVHHMEVFQCA $\bar{A} E S E D F P Q F N G P C D S K M K P D R L N Y C R$ & 300 \\
\hline & Rat & IMYEAIVTEGNEALVHHMEVFQCTNE ESEAFPMFNGPCDSKMKPDRLNYCR & 299 \\
\hline & Dog & 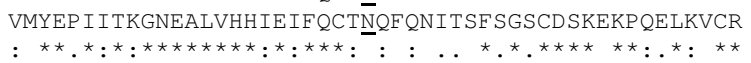 & 288 \\
\hline \multirow[t]{6}{*}{ HTR1A } & Human & KKSVNGESGSRNWRLGVESKAGGALCANGAVRQGDDGAALEVIEVHRVGN & 300 \\
\hline & Chimp & KKSVNGESGSRNWRLGVESK̄AGGALCANGAVRQGDDGAALEVIEVHRVGN & 300 \\
\hline & Dog & RKSVNGEP GGREWRQGPGSQ̄AGGPLCTNGAVRRGDDGAALEVIEVHRVGS & 300 \\
\hline & Mouse & KKSLNGQP GSGDCRRSAENRAVGTPCANGAVRQGEDDATLEVIEVHRVGN & 300 \\
\hline & Rat & KKSLNGQP GSGDWRRCAENR्̄AVGTPCTNGAVRQGDDEATLEVIEVHRVGN & 300 \\
\hline & & 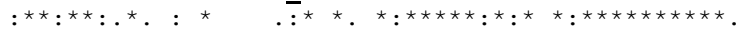 & \\
\hline \multirow[t]{6}{*}{ HTR1B } & Human & LLVMLLALITLATTLSNAFVIATVYRTRKLHTPANYLIASLAVTDLLVSI & 100 \\
\hline & Chimp & LLVM̄LLALITLATTLSNAFVIATVYRTRKLHTPANYLIASLAVTDLLVSI & 100 \\
\hline & Dog & LLVĪLLALITLATTLSNAFVIATVYRTRKLHTPANYLIASLAVTDLLVSI & 99 \\
\hline & Mouse & LLVE-LLALITLATTLSNAFVIATVYRTRKLHTPANYLIASLAVTDLLVSI & 96 \\
\hline & Rat & LLVÄLLALITLATTLSNAFVIATVYRTRKLHTPANYLIASLAVTDLLVSI & 96 \\
\hline & & **t* ${ }^{-}$- & \\
\hline \multirow[t]{6}{*}{ HTR1D } & Human & ATVLSNAFVLTTILLTRKLHTPANYLIGSLATTDLLVS ILVMP ISIA-YTI & 100 \\
\hline & Chimp & ATVLSNAFVLTTILLTRKLHTPANYLIGSLATTDLLVS ILVMP ISIĀYTI & 100 \\
\hline & Mouse & ATVLSNAFVLTTILLTKKLHTPANYLIGSLATTDLLVSILVMPISIĀYTT & 97 \\
\hline & Rat & ATVLSNAFVLTT I LLTKKLHTP ANYLI GSLATTDLLVS I LVMP ISI & 97 \\
\hline & Dog & ATALSNAFVLTT IFLTRKLHTPANYLIGSLAMTDLLVS I LVMP ISI & 100 \\
\hline & & 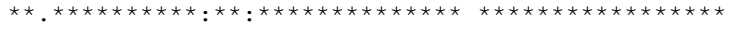 & \\
\hline \multirow[t]{6}{*}{ HTR1D } & Human & SSLCSLNSSLHEGHSHSAGSPLFFNHVKIKLADSALERKRISAARERKAT & 300 \\
\hline & Chimp & SSLCSLNSSLHEG̈HSHSAGSPLFFNHVKIKLADSALERKRISAARERKAT & 300 \\
\hline & Mouse & SSLCSLNPSLHE $\overline{\mathrm{S}} H T H T V G S P L F F N Q V K I K L A D S I L E R K R I S A A R E R K A T$ & 297 \\
\hline & Rat & SSLCSLNPSLHE & 297 \\
\hline & Dog & SSLCSLSP SLQEE ERSHAAGP PLFFNHVQVKLAEGVLERKRISAARERKAT & 300 \\
\hline & & 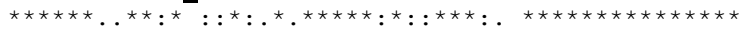 & \\
\hline \multirow[t]{6}{*}{ HTR2B } & Human & GNKLHWAALLILMVI IPT IGGNTLVILAVSLEKKLQYATNYF LMSLAVAD & 100 \\
\hline & Chimp & GNKLHWAALLILMVI IPTIGGNTLVILAVSLEKKLQYĀ̄NYFLMSLAVAD & 100 \\
\hline & Dog & GKKPQWAALLILTVI IP T I GGNI LVI LAISLEKKLQYĀTNYF LMS LAVAD & 99 \\
\hline & Mouse & GHTVHWAALLILAVI IPTIGGNI LVI LAVALEKRLQYĀ̄NYF LMSLAIAD & 99 \\
\hline & Rat & GNTVHWAALLIFAVI IPTIGGNI LVILAVSLEKRLQYĀT̄NYF LMS LAVAD & 99 \\
\hline & & 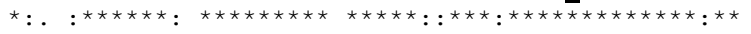 & \\
\hline \multirow[t]{6}{*}{ HTR2B } & Human & ATKSVKTLRKRSSKIYFRNPMAENSKFFKKHGIRNGINPAMYQSPMRLRS & 450 \\
\hline & Chimp & ATKSVKTLRKRSSKIYFRNPMAENSKFFKKHGIRNGINPAMYQSPMRLRS & 450 \\
\hline & Dog & AMKSVKTVRKCSSNNYFRN--GRELKVF HET-----WNV̄KWY-------- & 434 \\
\hline & Mouse & ATKSVKALRKFSSTLCFGNSMVENSKFFTKHGIRNGIND̄AMYQSPMRLRC & 449 \\
\hline & Rat & ATKSVKVLRKCSSTLYFGNSMVENSKFFTKHGIRNGINDAMYQSPVRLRS & 449 \\
\hline & & $\star \star \star \star \star .: \star \star \star \star . \quad \star * \quad .:{ }^{*} *:$ & \\
\hline \multirow[t]{6}{*}{ HTR3A } & Human & RKGVRPVRDWRKP TTVS IDVIVYAI LNVDEKNQVLTTYIWYRQYWTDEFL & 95 \\
\hline & Chimp & RKGVRPVRDWRKPTT̄IS IDVIVYAI LNVDEKNQVLTTYIWYRQHWTDEFL & 95 \\
\hline & Dog & QKGVRPVRDWRKPTĪVS IDVIVYAILSVDEKNQVLTTYIWYRQYWTDEFL & 100 \\
\hline & Mouse & KKGVRPVRDWRKPTT̄VS IDVIMYAI LNVDEKNQVLTTYIWYRQYWTDEFL & 99 \\
\hline & Rat & KKGVRPVRDWRKPT İVS IDV IMYAI LNVDEKNQVLTTY IWYRQFWTDEF L & 94 \\
\hline & & $-{ }^{-}$ & \\
\hline
\end{tabular}

Figure I

Alignment showing amino acid variation across five species in location of nsSNPs. The alignment show selected parts of the protein sequence containing the II detected nsSNPs in the dog, marked as bold and underlined residues. Only four species are aligned for the DRDI gene since there was no homologous gene available for the Chimpanzee (HomoloGene, [24]). 
primers were designed to amplify exonic sequence (primer 3, [34]).

The selected exonic sequences originated from a total of 18 genes, consisting of nine serotonin G protein-coupled receptors and one ligand-gated ion channel, three dopamine $\mathrm{G}$ protein-coupled receptors and additionally exons from four genes related to serotonin and dopamine formation and synaptic clearance. The study also included one enzymatic gene related to synthesis of norepinephrine (Table 1).

\section{PCR amplification and SNP detection}

Primers flanking each of the exon sequences of 12 serotonin-, five dopamine- and one norepinephrine-related genes (Table 1) were run in PCR with $\sim 25$ ng of canine genomic DNA as template, $1.5 \mu \mathrm{l} 10 \times$ PCR buffer containing $15 \mathrm{mM} \mathrm{MgCl}_{2}$ (Qiagen), $0.6 \mu \mathrm{ldNTP}(2.5 \mathrm{mM}), 0.5 \mu \mathrm{l}$ PCR primer, forward and reverse $(5 \mathrm{pmol} / \mu \mathrm{l}), 0.05 \mu \mathrm{l} \mathrm{Taq}$ DNA Polymerase ( $5 \mathrm{U} / \mu \mathrm{l}$, Qiagen) and water to a total volume of $15 \mu \mathrm{l}$. Initial denaturation at $95^{\circ} \mathrm{C}(21 / 2$ min.), followed by 34 cycles of $95^{\circ} \mathrm{C}\left(30 \mathrm{sec}\right.$ ), $58^{\circ} \mathrm{C}(40$ sec.), $72^{\circ} \mathrm{C}$ (50 sec.) and finally at $72^{\circ} \mathrm{C}(5 \mathrm{~min}$.). Primers not providing specific PCR products by these conditions were run at $95^{\circ} \mathrm{C}(21 / 2 \mathrm{~min}$.), followed by 34 cycles of $95^{\circ} \mathrm{C}(30 \mathrm{sec}$. $), 60^{\circ} \mathrm{C}\left(40 \mathrm{sec}\right.$ ), $72^{\circ} \mathrm{C}(50 \mathrm{sec}$.) and finally at $72^{\circ} \mathrm{C}(5 \mathrm{~min}$.).

The obtained PCR products were sequenced in both forward and reverse directions with the same PCR primers, by the MegaBACE ${ }^{\mathrm{rm}} 1000$ DNA Analysis Systems (Amersham Biosciences) using the DYEnamic ${ }^{\mathrm{Tm}}$ ET Dye Terminator Kit (Amersham Biosciences). Reaction conditions were as follows: $4 \mu \mathrm{l}$ ET reagent premix, $4.5 \mu \mathrm{H}_{2} \mathrm{O}, 1 \mu \mathrm{l}$ PCRproduct and $0.5 \mu \mathrm{l}$ primer $(5 \mu \mathrm{M})$ with the following step repeated 28 times: $95^{\circ} \mathrm{C}\left(15 \mathrm{sec}\right.$ ), $58^{\circ} \mathrm{C}\left(10 \mathrm{sec}\right.$.), $60^{\circ} \mathrm{C}$ ( $1 \mathrm{~min}$.). The post-reaction cleanup was performed as recommended by the protocol with ethanol and $7.5 \mathrm{M}$ ammonium acetate. SNPs were identified by aligning and comparing the sequence data with Sequencher 4.1.4 (Gene Codes Co.)

\section{SNP description and possible amino acid change}

Reference sequences were displayed from available databases and open reading frames (ORFs) defined. Further alignment and translation with Sequencher 4.1.4 (Gene Codes Co.) defined the codons and amino acid changes (Table 2). Alignment of protein sequences with nsSNPs (reference sequences in Table 2) for detection of conservation across species was performed with ClustalW [25]. Prediction of a possible damaging effect of the amino acid substitutions caused by the nsSNPs was performed with PolyPhen [4].

\section{Authors' contributions}

$\mathrm{JV}$ carried out the majority of the molecular genetic studies, performed the analysis and drafted the manuscript. FL conceived of the study, and participated in its design and coordination and helped to draft the manuscript. Both authors read and approved the final manuscript.

\section{Additional material}

\section{Additional file 1}

"Reported SNPs with flanking nucleotide sequences". SNPs, listed according to Table 2, with 200 bp flanking sequences.

Click here for file

[http://www.biomedcentral.com/content/supplementary/14712156-9-10-S1.pdf]

\section{Acknowledgements}

We would like to acknowledge Ewen Kirkness at TIGR for providing sequence information from the standard poodle genome $(I, 5 X)$ in an early phase of the project, and Aina $\mathrm{H}$. Rengmark for substantial help in the sequencing of SNPs. Project support from Division of Genetics, Department of Basic Sciences and Aquatic Medicine, Norwegian School of Veterinary Science, Oslo, Norway, and The Research Council of Norway, grant no. $138793 / 130$.

\section{References}

I. Savitz JB, Ramesar RS: Genetic variants implicated in personality: a review of the more promising candidates. Am J Med Genet B Neuropsychiatr Genet 2004, I 3 I:20-32.

2. Manuck SB, Flory JD, Ferrell RE, Dent KM, Mann JJ, Muldoon MF: Aggression and anger-related traits associated with a polymorphism of the tryptophan hydroxylase gene. Biol Psychiatry 1999, 45:603-614.

3. Brunner HG, Nelen M, Breakefield XO, Ropers HH, van Oost BA: Abnormal behavior associated with a point mutation in the structural gene for monoamine oxidase A. Science 1993. 262:578-580.

4. Ramensky V, Bork P, Sunyaev S: Human non-synonymous SNPs: server and survey. Nucleic Acids Res 2002, 30:3894-3900.

5. Kravitz EA: Serotonin and aggression: insights gained from a lobster model system and speculations on the role of amine neurons in a complex behavior. J Comp Physiol [A] 2000, I 86:22I-238.

6. Stevenson PA, Dyakonova V, Rillich J, Schildberger K: Octopamine and experience-dependent modulation of aggression in crickets. J Neurosci 2005, 25:|43|-|44I.

7. Overli O, Pottinger TG, Carrick TR, Overli E, Winberg S: Brain monoaminergic activity in rainbow trout selected for high and low stress responsiveness. Brain Behav Evol 200I, 57:214-224.

8. Feltenstein MW, Sufka KJ: Screening antidepressants in the chick separation-stress paradigm. Psychopharmacology (Berl) 2005, 181:153-159.

9. Wendland JR, Lesch KP, Newman TK, Timme A, Gachot-Neveu H, Thierry B, Suomi SJ: Differential functional variability of serotonin transporter and monoamine oxidase a genes in macaque species displaying contrasting levels of aggressionrelated behavior. Behav Genet 2006, 36: I63-I72.

10. Marino MD, Bourdelat-Parks BN, Cameron LL, Weinshenker D: Genetic reduction of noradrenergic function alters social memory and reduces aggression in mice. Behav Brain Res 2005, 16 I: 197-203.

II. Shih JC, Grimsby J, Chen K, Zhu QS: Structure and promoter organization of the human monoamine oxidase $A$ and $B$ genes. J Psychiatry Neurosci 1993, I 8:25-32. 
12. Chen K, Holschneider DP, Wu W, Rebrin I, Shih JC: A spontaneous point mutation produces monoamine oxidase A/B knock-out mice with greatly elevated monoamines and anxiety-like behavior. I Biol Chem 2004, 279:39645-39652.

13. Chen X, Wang X, O'Neill AF, Walsh D, Kendler KS: Variants in the catechol-o-methyltransferase (COMT) gene are associated with schizophrenia in Irish high-density families. Mol Psychiatry 2004, 9:962-967.

14. Curran S, Purcell S, Craig I, Asherson P, Sham P: The serotonin transporter gene as a QTL for ADHD. Am J Med Genet B Neuropsychiatr Genet 2005, I34:42-47.

15. Frank B, Niesler B, Nothen MM, Neidt H, Propping P, Bondy B, Rietschel $M$, Maier W, Albus M, Rappold G: Investigation of the human serotonin receptor gene HTR3B in bipolar affective and schizophrenic patients. Am J Med Genet B Neuropsychiat Genet 2004, I 3 I: | -5.

16. Maron E, Nikopensius T, Koks S, Altmae S, Heinaste E, Vabrit K, Tammekivi V, Hallast P, Koido K, Kurg A, Metspalu A, Vasar E, Vasar V Shlik J: Association study of $\mathbf{9 0}$ candidate gene polymorphisms in panic disorder. Psychiatr Genet 2005, I 5: I7-24.

17. Saudou F, Amara DA, Dierich A, LeMeur M, Ramboz S, Segu L, Buhot $\mathrm{MC}$, Hen R: Enhanced aggressive behavior in mice lacking 5HTIB receptor. Science 1994, 265(5 I 80): 1875-1878.

18. Brouillette JA, Andrew JR, Venta PJ: Estimate of nucleotide diversity in dogs with a pool-and-sequence method. Mamm Genome 2000, I I: I079-1086.

19. Masuda K, Hashizume C, Kikusui T, Takeuchi Y, Mori Y: Breed differences in genotype and allele frequency of catechol $O$ methyltransferase gene polymorphic regions in dogs. J Vet Med Sci 2004, 66:183-187.

20. van den Berg L, Kwant L, Hestand MS, van Oost BA, Leegwater PA Structure and variation of three canine genes involved in serotonin binding and transport: the serotonin receptor IA gene (htr I A), serotonin receptor 2A gene (htr2A), and serotonin transporter gene (slc6A4). J Hered 2005, 96:786-796.

21. Masuda K, Hashizume C, Ogata N, Kikusui T, Takeuchi Y, Mori Y: Sequencing of canine 5-hydroxytriptamine receptor (5HTR) IB, 2A, 2C genes and identification of polymorphisms in the 5-HTRIB gene. J Vet Med Sci 2004, 66:965-972.

22. van den Berg L, Versteeg SA, van Oost BA: Isolation and charac terization of the canine serotonin receptor IA gene (htr I A). J Hered 2003, 94:49-56.

23. van den Berg L, Imholz S, Versteeg SA, Leegwater PA, Zijlstra C, Bosma AA, van Oost BA: Isolation and characterization of the canine serotonin receptor IB gene (htrIB). Gene 2004 326: $131-139$.

24. HomoloGene: HomoloGene. 2008 [http://www.ncbi.nlm.nih.gov/]

25. ClustalW: ClustalW. 2008 [http://www.ebi.ac.uk/clustalw/]

26. Lee MA, Keane OM, Glass BC, Manley TR, Cullen NG, Dodds KG McCulloch AF, Morris CA, Schreiber M, Warren J, Zadissa A, Wilson T, McEwan JC: Establishment of a pipeline to analyse non-synonymous SNPs in Bos taurus. BMC Genomics 2006, 7:298.

27. Zhao Z, Fu YX, Hewett-Emmett D, Boerwinkle E: Investigating single nucleotide polymorphism (SNP) density in the human genome and its implications for molecular evolution. Gene 2003, 3 | 2:207-2 I3.

28. Kirkness EF, Bafna V, Halpern AL, Levy S, Remington K, Rusch DB Delcher AL, Pop M, Wang W, Fraser CM, Venter JC: The dog genome: survey sequencing and comparative analysis. Science 2003, 301:1898-1903.

29. Lindblad-Toh K, Wade CM, Mikkelsen TS, Karlsson EK, Jaffe DB, Kamal M, Clamp M, Chang JL, Kulbokas EJIII, Zody MC, Mauceli E, Xie X, Breen M, Wayne RK, Ostrander EA, Ponting CP, Galibert F, Smith DR, Delong PJ, Kirkness E, Alvarez P, Biagi T, Brockman W, Butler J, Chin CW, Cook A, Cuff J, Daly MJ, DeCaprio D, Gnerre S: Genome sequence, comparative analysis and haplotype structure of the domestic dog. Nature 2005, 438:803-8I9.

30. Sunyaev S, Ramensky V, Koch I, Lathe W III, Kondrashov AS, Bork P: Prediction of deleterious human alleles. Hum Mol Genet 200I, 10:591-597.

31. Jegga AG, Gowrisankar S, Chen J, Aronow BJ: PolyDoms: a whole genome database for the identification of non-synonymous coding SNPs with the potential to impact disease. Nucleic Acids Res 2007, 35:D700-D706.

32. $\mathrm{Ng}$ PC, Henikoff S: SIFT: Predicting amino acid changes that affect protein function. Nucleic Acids Res 2003, 3 I:38I2-38I4.
33. Sambrook J, Fitsch EF, Maniatis T: Molecular cloning: a laboratory manual Edited by: Fritsch EF and Maniatis T. Cold Spring Harbor, N.Y., Cold Spring Harbor Laboratory; 1989.

34. Primer3: Primer3. 2008 [http://frodo.wi.mit.edu/cgi-bin/primer3/ primer3 www.cgi].

35. OMIM: OMIM. www ncbi nlm nih gov/entrez/query fcgi?db=OMIM 2008 [http://www.ncbi.nlm.nih.gov/entrez/query.fcgi?db=OMIM].

36. dbSNP: dbSNP. 2008 [http://www.ncbi.nlm.nih.gov/SNP/ snp blastByOrg.coi

37. Lehninger AL: Principles of biochemistry Edited by: Nelson DL and Cox MM. New York, Worth; 1993.
Publish with Biomed Central and every scientist can read your work free of charge

"BioMed Central will be the most significant development for disseminating the results of biomedical research in our lifetime. "

Sir Paul Nurse, Cancer Research UK

Your research papers will be:

- available free of charge to the entire biomedical community

- peer reviewed and published immediately upon acceptance

- cited in PubMed and archived on PubMed Central

- yours - you keep the copyright

Submit your manuscript here:

http://www.biomedcentral.com/info/publishing_adv.asp 